\title{
A Sco protein among the hypothetical proteins of Bacillus lehensis G1: Its 3D macromolecular structure and association with Cytochrome $\mathrm{C}$ Oxidase
}

Soo Huei Tan', Yahaya M Normi ${ }^{*}$, Adam Thean Chor Leow ${ }^{1}$, Abu Bakar Salleh¹, Roghayeh Abedi Karjiban ${ }^{1,2}$, Abdul Munir Abdul Murad ${ }^{3}$, Nor Muhammad Mahadi ${ }^{4}$ and Mohd Basyaruddin Abdul Rahman 1,2,4

\begin{abstract}
Background: At least a quarter of any complete genome encodes for hypothetical proteins (HPs) which are largely non-similar to other known, well-characterized proteins. Predicting and solving their structures and functions is imperative to aid understanding of any given organism as a complete biological system. The present study highlights the primary effort to classify and cluster 1202 HPs of Bacillus lehensis G1 alkaliphile to serve as a platform to mine and select specific $\mathrm{HP}(\mathrm{s})$ to be studied further in greater detail.

Results: All HPs of B. lehensis G1 were grouped according to their predicted functions based on the presence of functional domains in their sequences. From the metal-binding group of HPs of the cluster, an HP termed Bleg1_2507 was discovered to contain a thioredoxin (Trx) domain and highly-conserved metal-binding ligands represented by Cys69, Cys73 and His159, similar to all prokaryotic and eukaryotic Sco proteins. The built 3D structure of Bleg1_2507 showed that it shared the $\beta a \beta a \beta \beta$ core structure of Trx-like proteins as well as three flanking $\beta$-sheets, a $3_{10}$-helix at the $\mathrm{N}$-terminus and a hairpin structure unique to Sco proteins. Docking simulations provided an interesting view of Bleg1_2507 in association with its putative cytochrome c oxidase subunit II (COXII) redox partner, Bleg1_2337, where the latter can be seen to hold its partner in an embrace, facilitated by hydrophobic and ionic interactions between the proteins. Although Bleg1_2507 shares relatively low sequence identity (47\%) to BsSco, interestingly, the predicted metal-binding residues of Bleg1_2507 i.e. Cys-69, Cys-73 and His-159 were located at flexible active loops similar to other Sco proteins across biological taxa. This highlights structural conservation of Sco despite their various functions in prokaryotes and eukaryotes.

Conclusions: We propose that HP Bleg1_2507 is a Sco protein which is able to interact with COXII, its redox partner and therefore, may possess metallochaperone and redox functions similar to other documented bacterial Sco proteins. It is hoped that this scientific effort will help to spur the search for other physiologically relevant proteins among the so-called "orphan" proteins of any given organism.
\end{abstract}

Keywords: Hypothetical proteins, Bleg1_2507, Sco, Thioredoxin, Copper binding, Redox reaction, Cytochrome c oxidase

\footnotetext{
* Correspondence: normi_yahaya@upm.edu.my

${ }^{1}$ Center for Enzyme and Microbial Biotechnology (EMTECH), Faculty of

Biotechnology and Biomolecular Sciences, Universiti Putra Malaysia, Serdang,

Selangor 43400, Malaysia

Full list of author information is available at the end of the article
}

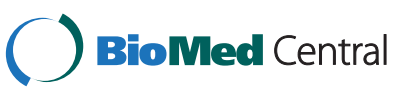

(c) 2014 Tan et al.; licensee BioMed Central Ltd. This is an Open Access article distributed under the terms of the Creative Commons Attribution License (http://creativecommons.org/licenses/by/2.0), which permits unrestricted use, distribution, and reproduction in any medium, provided the original work is properly credited. The Creative Commons Public Domain Dedication waiver (http://creativecommons.org/publicdomain/zero/1.0/) applies to the data made available in this article unless otherwise stated. 


\section{Background}

Hypothetical proteins (HPs) are generally known as proteins of unknown structures and functions [1]. They constitute approximately $25 \%$ of any sequenced genome even in simple, model organisms such as Escherichia coli, Bacillus subtilis and Saccharomyces cerevisiae [2]. Annotating their biological function has remained a challenge due to their low sequence and structural homology to other known proteins [3]. This in turn creates difficulties in attempts to understand a biological system as a whole due to the information gaps posed by these "orphan" proteins. Therefore, efforts in trying to unravel their structures and functions are crucial in filling these information gaps for any particular system and hence, should be encouraged. To deepen our understanding on these proteins, in silico methods of analysis are the most time and cost effective in generating a wealth of reliable and useful information on HPs ranging from their localization, properties to their possible structures and functions. It also provides a quick means to preliminary screen and chooses potential HPs for subsequent downstream in vitro and in vivo experiments, particularly to initiate structural genomic projects in the future [4].

With the wealth of information which can be generated using the in silico approach, this present study highlights the utilization of various in silico tools and methods to functionally predict and cluster all HPs present in the complete genome of a locally isolated alkaliphile, Bacillus lehensis G1, with the purpose of establishing a platform to mine and later select specific HPs to be studied further in greater detail. Whilst the genome of this alkaliphile has been completely sequenced [5], the structural and functional omics of this extremophile is not well characterised, particularly its HPs. Hence, our attempt at firstly predicting their functions and clustering them accordingly will serve as a platform in mining and selecting specific HPs to be studied further in greater detail. Stemming from this effort, our attention was led to a particular HP, Bleg1_2507, in the metalloprotein category of the cluster. This HP contained a Sco1 domain of the Thioredoxin (Trx) superfamily and showed up to $50 \%$ of sequence identity to other bacterial HPs and $47 \%$ to bacterial Sco proteins.

It is important to note that Sco proteins are present in both prokaryotic and eukaryotic organisms and are required for the proper assembly of cytochrome c oxidase (COX), a terminal enzyme in the respiratory chain [6]. Sco was suggested to be involved in the delivery of copper ion to COX complex [7-9]. To date, the only bacterial Sco1 protein which was structurally studied and analysed at length is from Bacillus subtilis [6] while its eukaryotic counterpart is from yeast [10], human [11] and plant [12]. Sco proteins have garnered importance in recent years due to their roles in the correct assembly of the copper center $(\mathrm{CuA})$ of COX subunit II (COX II).
Improper assembly of COX has been reported to cause fatal infantile encephalopathy due to mutations in Sco1 and $S c o 2$ genes in humans $[11,13]$.

Due to the physiological relevance of Sco proteins, we embarked on the task of building the structure of HP Bleg1_2507 of B. lehensis G1 via homology modelling to investigate the structural similarities and differences between this protein with Scol protein from B. subtilis (BsSco), yeast and human. As Sco1 was suggested to be involved in the delivery of copper ion to COX complex, specifically COXII [7-9], we subsequently mined the genome of $B$. lehensis $\mathrm{G} 1$ for the sequence encoding COXII by performing a BLASTP search. As a result, Bleg1_2337 was retrieved and its structure was built via homology modelling. Docking of both HP Bleg1_2507 and Bleg1_2337 models was performed to investigate their possible interaction. Based on the results obtained, the possible structure, function and mechanism of HP Bleg1_2507 from B. lehensis G1 are duly discussed. Lastly, the structure of HP Bleg1_2507 of B. lehensis G1 was compared to other Sco proteins to highlight its similarity and distinctness.

\section{Methods}

Domain and sequence similarity analysis of HPs of $B$. lehensis G1: Development of an HP cluster

All sequences encoding HPs in the genome of B. lehensis G1 were firstly subjected to INTERPROSCAN [14] and Conserved Domain Search (NCBI-CDD) [15] analyses to detect possible functional domains within the sequences. Sequence similarity of all the HPs with other proteins in the database was investigated also using BLASTP [16]. Based on the predicted functional domains and sequence similarity, an HP cluster was built to categorize the proteins accordingly. A scan on HPs which showed acceptable similarity to other proteins with low e-value was carried out and the potential candidate was chosen for further analyses and structure prediction.

\section{Sequence analysis of selected HP (Bleg1_2507) and putative Cytochrome c Oxidase subunit II, COXII (Bleg1_2337)}

From the above scan, a particular HP in the metal-binding group of proteins encoded by bleg1_2507 gene was chosen for further analyses based on the presence of the conserved copper chaperone Sco1 domain related to Trx-like superfamily. Firstly, the possible presence of consensus amino acids pattern in Bleg1_2507 sequence was identified using ScanProsite [17]. Important and metal-binding residues were subsequently identified using Consurf [18] and MetalDetector v 2.0 [19] respectively. The physicochemical aspect of the protein such as its theoretical pI value was investigated using ProtParam [20]. SigCleave from EMBOSS [21] was used to investigate the possible presence of 
signal peptide in Bleg1_2507, while the evolutionary relatedness of the protein with other known proteins was investigated using PHYLIP [22].

Subsequently, a genome-wide scan for Bleg1_2507 redox partner i.e. cytochrome c oxidase subunit II (COX II), was performed. As a result, Bleg1_2337, a putative COXII protein was identified from the genome sequence of B. lehensis G1. Further analyses similar to the ones mentioned above were performed on Bleg1_2337.

\section{Homology modeling of HP Bleg1_2507 and Bleg1_2337}

PSI-BLAST [16] search against Protein Data Bank (PDB) was performed to retrieve potential templates for Bleg1_2507 and Bleg1_2337 model construction. Subsequently, Multiple Sequence Alignment (MSA) of the templates with the query sequences was performed using ClustalW [23] to determine the degree of similarity and conservation of specific motifs and amino acids in the sequences. $3 \mathrm{D}$ models of both proteins were developed via homology modeling using MODELLER 9v10 [24]. The best built models for Bleg1_2507 and Bleg1_2337 were chosen based on their lowest Discrete Optimized Protein Energy (DOPE) values and GA 341 score of one, which signify that the models resemble the native structure and hence, reliable. The generated models were visualized by PYMOL [25]. To aid assessment and ease discussion on the models, a color gradient scheme representing different levels of hydrophobicity of the amino acid side chains [26] were utilized. Further confirmation on the validity of the models was made based on their Root Mean Square Deviation (RMSD) calculated by superimposing them with their respective templates using Chimera USCF 1.6.1 [27].

\section{Model refinement and validation}

Refinement of the built models was performed using FoldX [28] (RepairPDB) to remove Van Der Waals clashes and bad contacts of amino acid side chains. Subsequently, structural evaluation and stereochemical analyses of the refined models were performed using ERRAT which sets a 95\% confidence limit being the cut-off value to evaluate any incorrect residues present in the protein structure based on the average of six atomic interactions in the protein i.e. $f$ (CC), $f(\mathrm{CN}), f(\mathrm{CO}), f(\mathrm{NN}), f(\mathrm{NO})$ and $f(\mathrm{OO})$ [29]. Further supporting analysis to evaluate the models was performed using PROCHECK Ramachandran plot [30].

\section{Docking of Bleg1_2507 and Bleg1_2337 models and energy minimization of protein complex}

Both of the models were docked by using Cluspro v 2.0 [31] to predict protein interaction. Subsequently, the docked protein complex was refined using AMBER force in YASARA [32].

\section{Results and discussion}

Domain and sequence similarity analyses and clustering of HPs of B. lehensis $\mathrm{G} 1$

Based on the BleG1DB v1.0 complete genome of the locally isolated alkaliphilic B. lehensis G1 (released Aug 24th, 2012), the 3.5 Mbp genome consisted of 4021 predicted genes, in which 2819 of them encode proteins with putative functions while 1202 of them encode uncharacterized, hypothetical proteins [http://27.126.156.144/]. INTERPROSCAN analysis on all the HPs revealed that $51.5 \%$ of them were predicted with functions, while $48.5 \%$ were of unknown functions and hence categorized as unknown proteins (Table 1). In the category of proteins with predicted functions, majority of the HPs (286 in total) were signal peptides, in which 221 of them were transmembrane peptides. Other than these signal peptides, there were other 47 transmembrane proteins as well. The second most abundant type of HPs (totaling up to 115) was predicted to have enzymatic functions. The remaining numbers of HPs were predicted to be regulatory proteins (52), metal-binding proteins (43) and antibiotic-related (12) (Table 1).

\section{Bleg1_2507 and Bleg1_2337 (putative COX II) Sequence Analyses}

Within the pool of metal-binding proteins, HP Bleg1_2507 which consists of 198 amino acids was discovered to possess a copper chaperone Sco1 domain related to Trx-like superfamily from both INTERPROSCAN and NCBI-CDD analyses (data not shown).

Sequence similarity analysis of the HP using BLASTP indicated that Bleg1_2507 is similar to HPs of other $\mathrm{Ba}$ cillus species with 40-50\% identities (Table 2). Additionally in the top 10 hits of the BLASTP analysis, results

Table 1 Number of hypothetical proteins based on predicted functions

\begin{tabular}{lll}
\hline Predicted functions & No. of HPs & Percentage \% \\
\hline Unknown protein functions & 583 & 48.5 \\
Signal peptide, transmembrane & 221 & 18.3 \\
Enzymes & 115 & 9.5 \\
Signal peptides & 65 & 5.4 \\
Regulatory proteins & 52 & 4.3 \\
Transmembranes & 47 & 3.9 \\
Metal-binding proteins & 43 & 3.6 \\
Lipoproteins & 27 & 2.2 \\
Transporters & 20 & 1.7 \\
Antibiotic-related proteins & 12 & 1.0 \\
Spore proteins & 9 & 0.7 \\
Others & 8 & 0.7 \\
Total & 1202 & 100.0 \\
\hline
\end{tabular}


Table 2 Top 10 hits of BLASTP similarity search against NR database for Bleg1_2507

\begin{tabular}{llcc}
\hline Accession No. & Description & Identity \% & E-value \\
\hline NP 244201.1 & Hypothetical Protein BH 3335 (Bacillus halodurans C-125) & 50 & $2 \mathrm{e}-63$ \\
YP 176895.1 & Hypothetical Protein ABC 3401 (Bacillus clausii KSM-K16) & 47 & $1 \mathrm{e}-43$ \\
YP 003425399.1 & Unnamed protein product (Bacillus pseudofirmus OF4) & 45 & $3 \mathrm{e}-61$ \\
YP 173730.1 & Hypothetical Protein ABC 0226 (Bacillus clausii KSM-K16) & 36 \\
NP 243770.1 & Hypothetical Protein BH 2904 (Bacillus halodurans C-125) & 40 \\
EIM 07312.1 & Electron transport protein SCO1/SenC (Planococcus antarcticus DSM 14505) & 47 \\
ZP 08680789.1 & Sco1 family electron transport protein (Sporosarcina newyorkensis 2681) & 42 \\
EIJ 83190.1 & Electron transport protein SCO1/SenC (Bacillus methanolicus MGA3) & 38 \\
ZP 10043231.1 & Sco1/SenC (Bacillus sp.5B6) & 35 \\
YP 003920672.1 & Assembly factor BSco of the CuA site of cytochrome c oxidase (Bacillus amyloliquefaciens DSM7) & 35 \\
\hline
\end{tabular}

also indicated that Bleg1_2507 shared similarity to electron transport protein Sco1 of various microorganisms. Sco1/SenC protein from Planococcus antarcticus DSM 14505 has the highest identity of $47 \%$ while the model Sco1 protein from B. subtilis (BsSco) surprisingly shared only 35\% identity with Bleg1_2507 (Table 2).

Further analysis on Bleg1_2507 using ScanProsite revealed that amino acids 31-196 matched a thioredoxin_2 consensus pattern with total score of 10. Apart from this, analysis on the degree of amino acids conservation in Bleg1_2507 using Consurf revealed that Cys-69, Cys-73 and His-159 were highly conserved and similar to the ones in the top 10 hits from the UniRef 90 database of the Consurf program. The hits include the assembly factor of copper II (CuA) site of COX in B. atrophaeus 1942, Sco1 protein homolog from B. subtilis (BsSco) and putative uncharacterized protein from B. clausii KSM-16. In addition to this, these residues were also identified as potential metal-binding ligands via MetalDetector v2.0 analysis. These metal-binding residues resemble those of Sco1 proteins which similarly contain two Cys and a His residues at their metal-binding sites [33]. Hence, there is a possibility that the conserved Cys-69, Cys-73 and His-159 residues of Bleg1_2507 form the metal binding site of Bleg1_2507. Further analysis on the sequence of Bleg1_2507 revealed that the protein also possessed CXXXC and DXXXD motifs, which are very well preserved in all eukaryotic and prokaryotic Sco proteins [34,35]. Both of the conserved Cys in the CXXXC motif of Bleg1_2507 are made up of Cys-69 and Cys-73 respectively while the conserved Asp in the DXXXD motif are made up of Asp-102 and Asp-106 respectively (Figure 1). The conserved Asp residues of the motif have been implicated in copper ion binding during $\mathrm{CuA}$ assembly [35].

Since the results above highlight the possibility of $\mathrm{HP}$ Bleg1_2507 to be a Sco1 protein, a genome search for the sequence encoding the well-documented redox partner of
Sco1, COXII [35] was performed. According to [35], 82\% of regular prokaryotic genomes possessed the same number of sco and coxII genes or the absence of both. Based on BLASTP result of Bleg1_2507, there were 5 HPs which exhibited $40-50 \%$ of sequence similarity to Bleg1_2507, originating from $B$. halodurans, $B$. clausii KSM-K16 and $B$. pseudofirmus OF4 (Table 2). Search for COXII from the genomes of these microorganisms revealed that COXII was present in these microorganisms with the accession number of NP_243481.1, YP_175889.1 and YP_003425144.1 respectively. This suggested a correlation between these genes and highlights the possible presence of the coxII gene in the genome of B. lehensis G1. The genome search led to the retrieval of Bleg1_2337 sequence whereby BLASTP analysis on this 344 amino acids protein showed $80 \%$ identity to COXII of B. clausii KSM-16. ScanProsite analysis showed the presence of Cox2_CuA (PS 50857) domain in Bleg1_2337 spanning from amino acid number 125-236. Three conserved amino acid residues namely Cys-261, Cys-264 and His-265 were identified to be metal-binding ligands. In addition to these residues, Metal Detector v2.0 program predicted Cys-207, Cys-211 and His-215 to be metal-binding ligands as well. ClustalW alignment of Bleg1_2337 showed that its predicted metal-binding residues, Cys-207, Cys-211 and His-215 are very well aligned with those of COXII sequences from other organisms such as Thermus thermophiles, Bos taurus and Paracoccus denitrificans (Figure 2). This result strengthens the prediction that Bleg1_2337 is indeed a COXII protein and that the sets of conserved residues are indeed metal-binding ligands, similar with reported metal-binding ligands of other COXII proteins [36].

Further probing on Bleg1_2337 using Consurf indicated that several hydrophobic residues such as Val-170, Ser-173, Phe-174, Trp-175 and Pro-177 were found to be highly conserved as well (Figure 2). Such hydrophobic residues have been highlighted to play an important role in the hydrophobic interaction with Sco protein [37]. 


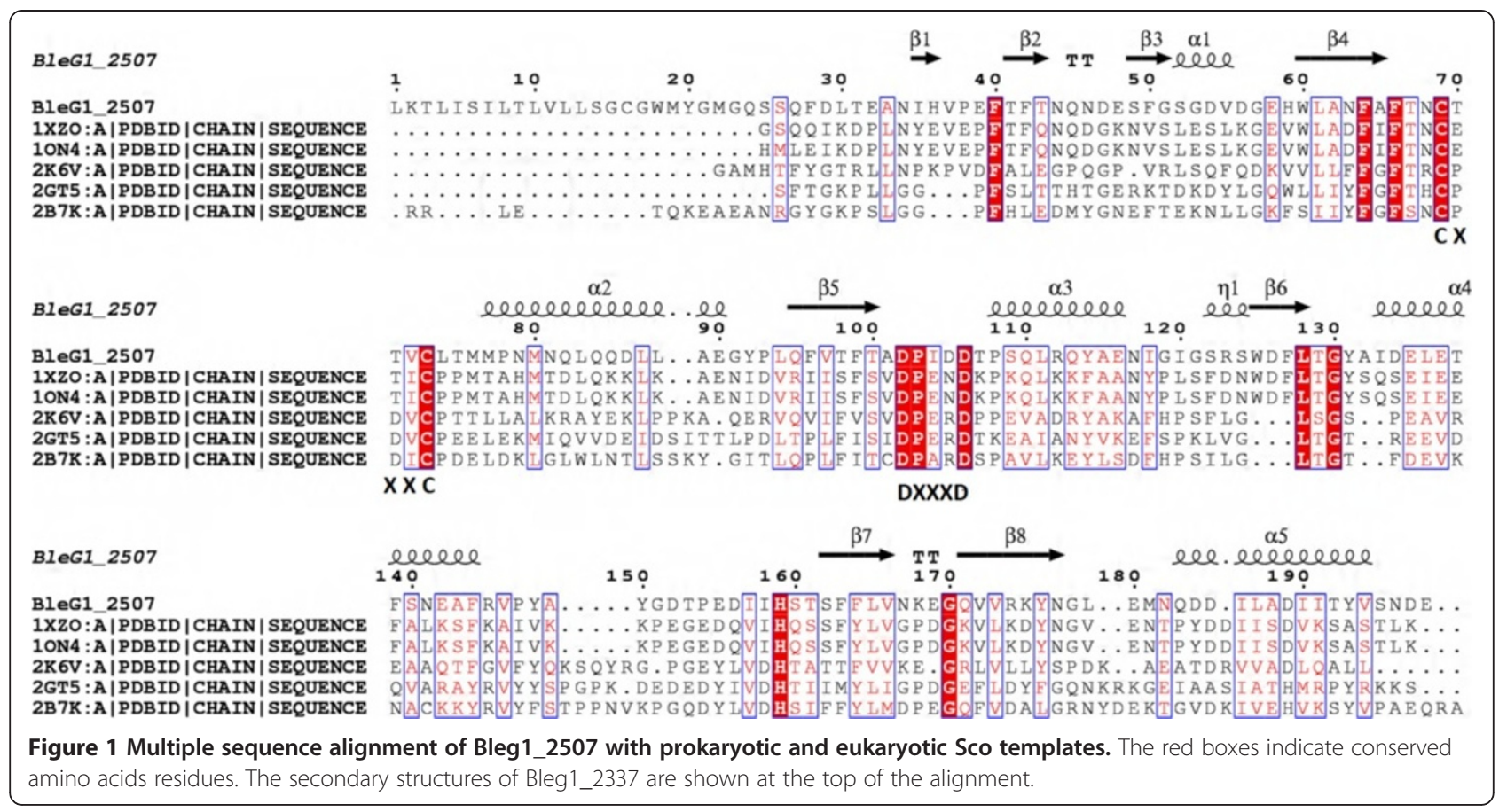

BLEG1_2337

BLEG1_2337

1AR1 : B |PDBID | CHAIN | SEQUENCE 1CYX:A|PDBID | CHAIN | SEQUENCE 1FFT:B | PDBID | CHAIN | SEQUENCE $1 \mathrm{M} 56$ : B|PDBID | CHAIN | SEQUENCE 1V54 : B | PDBID | CHAIN | SEQUENCE 2YEV : B | PDBID | CHAIN | SEQUENCE

BLEG1_2337

BLEG1_2337

1AR1 : B |PDBID | CHAIN | SEQUENCE 1CYX:A|PDBID | CHAIN | SEQUENCE 1FFT : B | PDBID | CHAIN | SEQUENCE 1 M56: B PDBID | CHAIN | SEQUENCE $1 \mathrm{~V} 54$ : B | PDBID | CHAIN | SEQUENCE 2YEV : B | PDBID | CHAIN | SEQUENCE

BLEG1_2337

BLEG1_2337

1AR1: B PDBID | CHAIN | SEQUENCE 1CYX:A|PDBID | CHAIN | SEQUENCE 1FFT : B | PDBID | CHAIN | SEQUENCE 1 56 : B | PDBID | CHAIN | SEQUENCE $1 \mathrm{~V} 54$ : B|PDBID | CHAIN | SEQUENCE 2YEV : B|PDBID | CHAIN | SEQUENCE

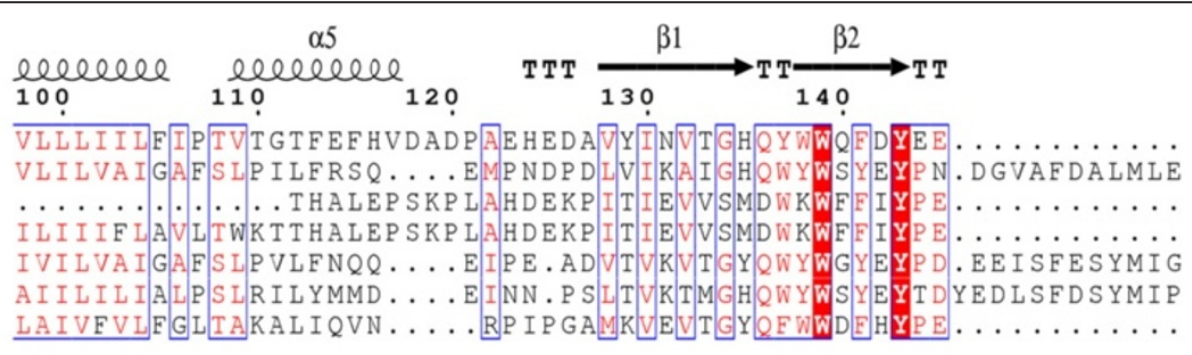

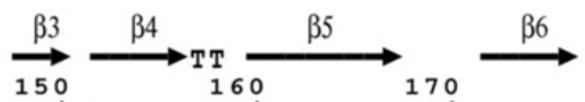

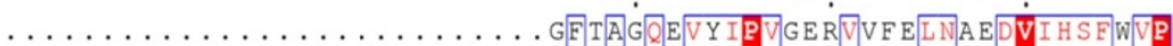

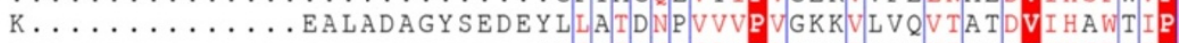

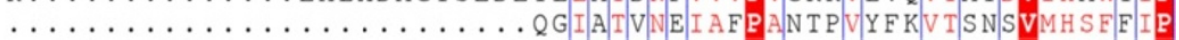
$\ldots \ldots \ldots \ldots \ldots \ldots \ldots \ldots \ldots \ldots$ GIATVNEIAFPANT PVYFKVTSNSVMNSEFIP SPATGGDNRMSPEVEQLLIEAGYSRDELLATDIAMVVPVNKTVVVQVTGADVIHSWTVE

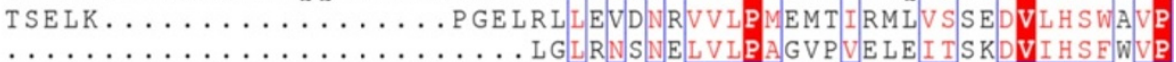

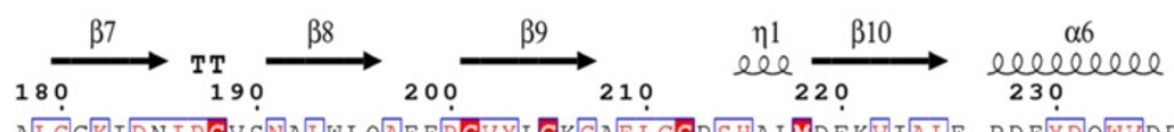

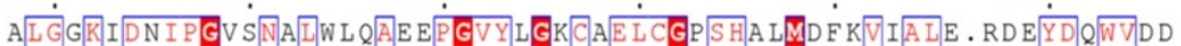

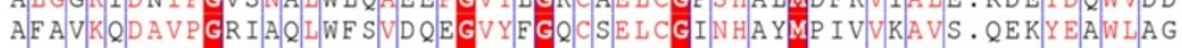

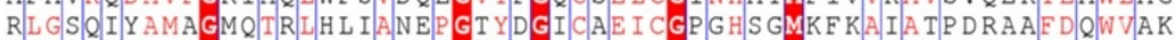

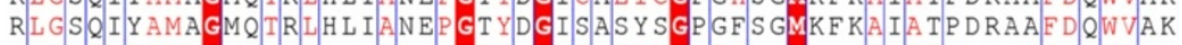

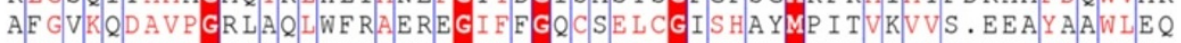

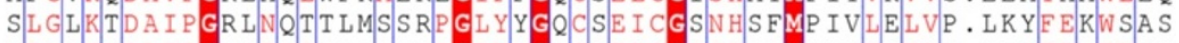

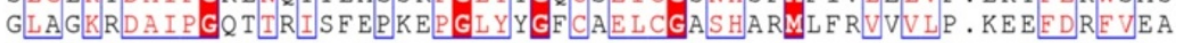

\section{CXXXC}

Figure 2 Multiple sequence alignment of Bleg1_2337. Yellow boxes indicate the conserved amino acid residues, while red boxes indicate metal-binding residues. 
Homology modeling of Bleg1_2507 and Bleg1_2337

Potential templates for homology modeling of both Bleg1_2507 and Bleg1_2337 were retrieved using PSIBLAST [16] search against PDB. Four templates were obtained for HP Bleg1_2507. Alignment of these sequences with Bleg1_2507 showed that Cys-69, Cys-73 of the CXXXC motif, His-159, Asp-102 and Asp-106 of the DXXXD motif of the HP aligned perfectly with all of the potential templates (Figure 1). 1XZO, chain A, which is a crystal structure of a disulfide switch in B. subtilis Sco (BsSco) and a well-studied member of the Sco family of COX assembly proteins [37] was selected as the template for homology modeling as it possessed the highest sequence similarity to Bleg1_2507 with 39\% identity and lower e-value of $5 \mathrm{e}-31$ compared to other templates (data not shown). In addition to this, parsimony phylogenetic analysis from PHYLIP also revealed that $1 \mathrm{XZO}$ is closely related to Bleg1_2507 (Figure 3(A)), lending further credence for it to be used as the template for Bleg1_2507 model generation.

As for Bleg1_2337, six templates were obtained and alignment of their sequences with Bleg1_2337 showed that highly the conserved residues, Cys-207, 211 and His-215 as well as the hydrophobic residues mentioned above were very well aligned. 2yev chain $B$, which is a crystal structure of caa3-type cytochrome oxidase Thermus thermophilus HB8 [36] was selected as the template for homology modeling of Bleg1_2337 as it has the highest sequence identity of $37 \%$ and low e-value of $4 \mathrm{e}-50$ when compared to other templates. Phylogenetic analysis from PHYLIP also revealed that 2YEV chain $B$ is closely related to Bleg1_2337 (Figure 3(B)).

The best built models for Bleg1_2507 and Bleg1_2337 (Figure 4(A) \& (C)) have the lowest DOPE values and GA 341 scores of one, suggesting that the developed models resemble the native structure and hence reliable. Further supporting results from the superimposition of all $\mathrm{C} \alpha$ atoms of both developed models and templates gave forth low RMSD of less than $0.5 \AA$, suggesting that the built models are indeed reliable.

\section{Bleg1_2507 and Bleg1_2337 model refinement and validation}

Refinement of the built models using FoldX in YASARA resulted in minimization of the free energy of Bleg1_2507 from $296.2 \mathrm{kcal} / \mathrm{mol}$ to $62.2 \mathrm{kcal} / \mathrm{mol}$ and from $657.5 \mathrm{kcal} /$ $\mathrm{mol}$ to $247.1 \mathrm{kcal} / \mathrm{mol}$ for Bleg1_2337.

Evaluations on the quality of the refined protein structures using ERRAT [29] indicated that $84.2 \%$ of the amino acids of the refined Bleg1_2507 model were located in the acceptable region within $95 \%$ confidence limit, as opposed to only $70.5 \%$ before refinement. As for refined Bleg1_2337 model, $85.4 \%$ of its amino acids were located in the acceptable region within the $95 \%$ confidence limit, as opposed to only $75.4 \%$ before refinement. These indicated that the refined protein models for Bleg1_2507 and Bleg1_2337 (Figure 4(A) \& (C)) were precise and reliable since the frequencies of atom randomizations were low. Further supporting results from PROCHECK Ramachandran analysis on refined Bleg1_2507 model revealed that $85.7 \%$ of the residues were located in favored region, $12.3 \%$ in additional allowed region, $1.3 \%$ and $0.6 \%$ in generously allowed and disallowed regions respectively. As for Bleg1_2337 refined model, $87.7 \%$ of the residues were located in favored region, $10.3 \%$ in additional allowed region and $2 \%$ were located in generously allowed region. Overall, both ERRAT and PROCHECK evaluations further reaffirmed that both of the predicted protein models were reliable.

\section{Probing the structures of Bleg1_2507 and Bleg1_2337}

Overall, the built model for HP Bleg1_2507 showed that it adopted a global topology unique to Thioredoxin (Trx)-like superfamily of proteins including Sco proteins whereby four $\beta$-sheets $(\beta 4, \beta 5, \beta 7$ and $\beta 8)$ are flanked by three $\alpha$-helices $(\alpha 1, \alpha 3$ and $\alpha 4)$ at the core of the structure (Figure 4(A)). This is similar to Trx-like superfamily members which have a characteristic, common core $\beta \alpha \beta \alpha \beta \beta$ secondary structural pattern with different insertions of secondary structural elements to distinguish the various structural families such as thioltransferases,

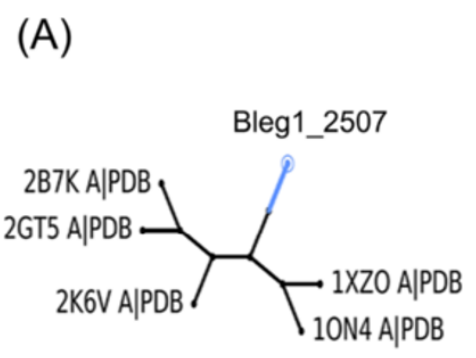

(B)

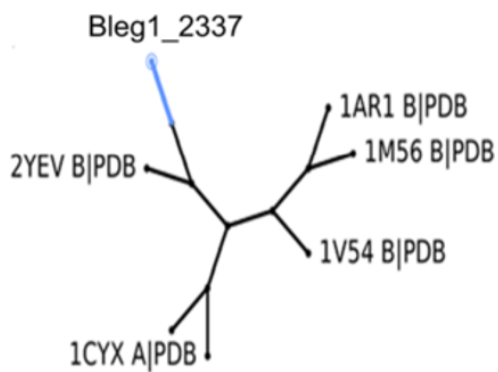

Figure 3 Phylogenetic analysis of (A) Bleg1_2507 and (B) Bleg1_2337 with their possible structural templates. 


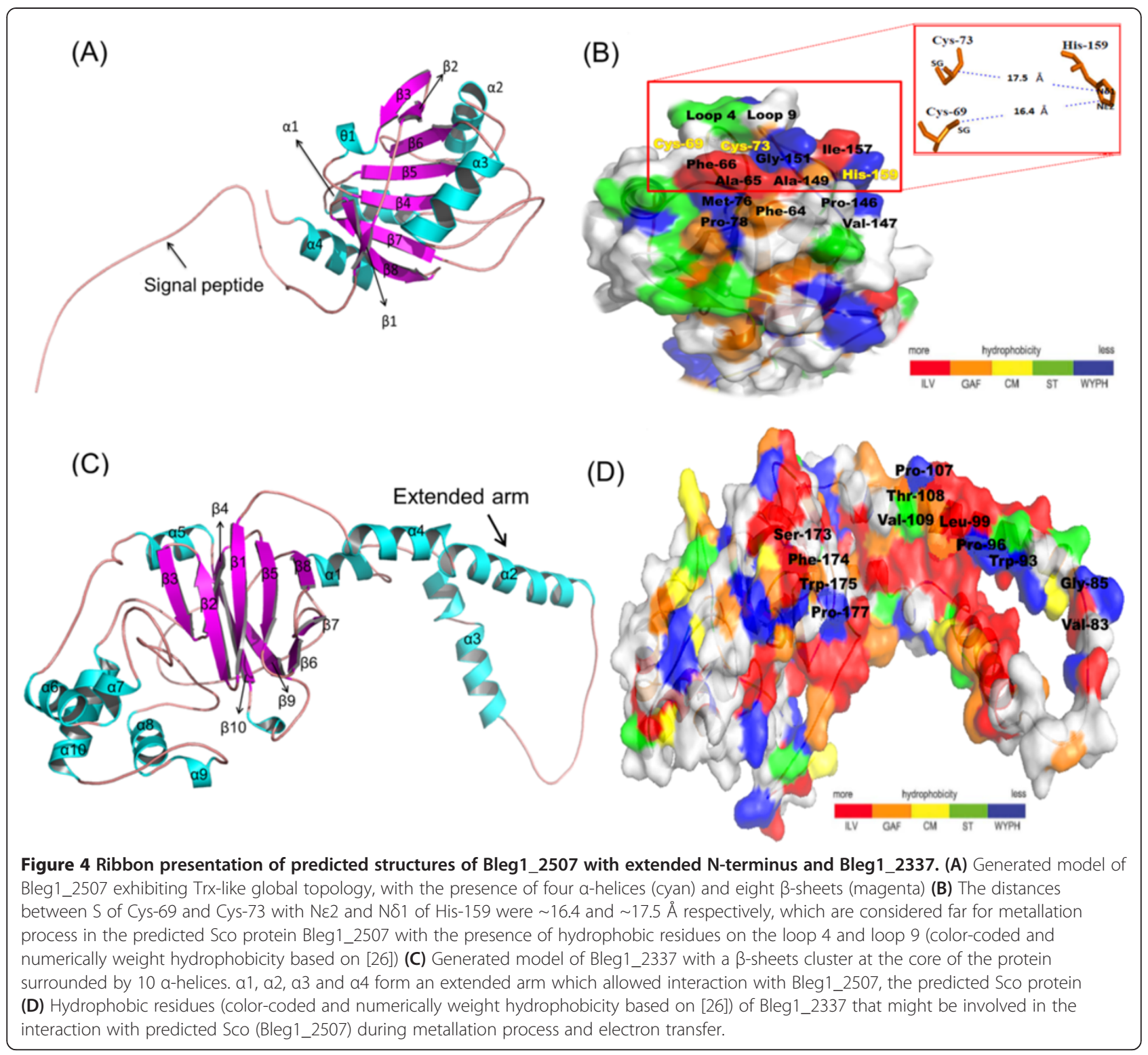

glutaredoxins, bacterial arsenate reductases and disulfide bond isomerases. They are in general involved in cellular thiol-redox reactions to maintain the reduction and oxidation of proteins or certain small molecules [38]. Other than this core structure, Bleg1_2507 also consisted three flanking $\beta$-sheets ( $\beta 1, \beta 2$ and $\beta 3$ ), a $3_{10}$-helix at the $\mathrm{N}$ terminus and a hairpin formed by $\beta 2$ and $\beta 3$, which are the additional secondary structure elements only present in Sco proteins [39].

Other similarities between include the presence of a transmembrane region in the N-terminal of Bleg1_2507 in which an external loop comprising of 25 amino acids at the $\mathrm{N}$-terminus could be observed (Figure 4(A)). SigCleave analysis from EMBOSS [21] revealed that the amino acids from Leu-4 to Cys-16 in this loop were predicted to be a signal peptide, which can be subjected to cleavage to give forth the mature protein. Previous study has shown that in the Sco protein of B. subtilis, (BsSco) the first 20 amino acids are located in the transmembrane and Cys-16 is the signal peptidase II recognition site. To this cleaved site a diacyl glycerol moiety will be attached to the protein and the post-translationally modified protein will subsequently be able to anchor to the membrane through the attached lipid by covalent bond [39]. This in turn highlights that Cys-16 in HP Bleg1_2507 is most probably a cleavage recognition site similar to the one found in BsSco for processing of the native Sco protein before it can be partially integrated into the membrane.

Unique to Bleg1_2507 however, there is an $\alpha$-helix $(\alpha 2)$ near loop 4 and a $\beta$-strand ( $\beta 6)$ that are parallel with $\beta 5$ and $\beta 4$ (Figure $4(\mathrm{~A})$ ), which are not present in 
Trx. In addition to this, the location of the metalbinding ligands in Bleg1_2507 is different from the solution structure of BsSco. For instance, in the predicted model of Bleg1_2507, both metal-binding Cys-69 and Cys-73 residues were located in loop 4 (situated in between $\alpha 1$ and $\beta 4$ ) while His-159 was positioned in loop 9 (situated in between $\alpha 3$ and $\beta 7$ ) (Figure 4(B)). In BsSco on the other hand, its metal-binding residues, namely Cys-45 and Cys-49, were located in loop 3 and the conserved His-135 was located in loop 8 [6].

Looking closely at the predicted metal-binding cavity of Bleg1_2507, it featured the highly conserved Cys-69, Cys73 (of the CXXXC motif) and His-159 residues. These residues which are strictly preserved in all Sco proteins have been implicated to be responsible in copper-binding and redox reaction [39]. In proximity to the CXXXC motif, a hydrophobic groove which is formed by Phe-64, 66, Ala-65, Val-72, Met-76, 77 and Pro-78 was observed (Figure 4(B)). Besides this, another hydrophobic groove formed by the His-ligand loop consisting of Val-146, Pro147, 154, Ala-149, Gly-151, Ile-157, 158, and Phe-163 was also observed (Figure 4(B)). Both of the hydrophobic grooves formed a hydrophobic pocket which may allow proteins and small molecules such as copper ion to be accommodated [6].

According to Balatri et al., 2003 [6], the interaction of hydrophobic residues within the protein are essential in the metallated state of Sco, because it is able to stabilize the metal-ligand region of the protein, which are located far apart from each other within the two hydrophobic grooves, by coordinating the metal-ligand geometry. Based on the Sco1 protein crystal structures from $B$. subtilis [6], yeast [10] and human [40] the distances between the $\mathrm{N}$ atoms of His residues and the $\mathrm{S}$ atom of Cysteine pair are in the range of 10-19 $\AA$. In the case of Bleg1_2507, the distance between Nع2 of His-159 and S of Cys-69 was $16.4 \AA$, while the distance between $N \delta 1$ of His-159 and S of Cys-73 was $17.5 \AA$ (Figure 4(B)), similar to the range noted above for the respective Sco1 proteins from B. subtilis, yeast and human. These distances are considered far for metal-ligand interaction in the apo-form state of the predicted Sco protein. It has been proposed by Balatri et al., 2003 [6] that the hydrophobic residues in the hydrophobic grooves of Bleg1_2507 might be responsible in regulating the appropriate geometric coordination for metal-ligand interaction to occur. According to Andruzzi et al. [41], the oxidized BsSco was coordinated to $\mathrm{Cu}$ (II) by a distorted tetragonal square planar, in which the Cys-45, Cys-49 and His-135 are ligated equatorially and water was ligated axially to the $\mathrm{Cu}$ (II) respectively. Hence, Cys-69, Cys-73 and His-159 of the predicted Sco of Bleg1_2507 was proposed to coordinate the copper through square planar geometry. Based on these observations, we propose that this particular HP is potentially a Sco protein with an ability to bind and chaperone copper to the CuA site in COXII.

\section{Interaction between Bleg1_2507 and putative COXII redox protein, Bleg1_2337 and their possible mechanism of action}

To test the above hypothesis, HP Bleg1_2507 was docked to its predicted redox partner, Bleg1_2337, a putative COXII protein to investigate possible interaction between these two proteins. Docking results showed that Bleg1_2507 was indeed able to interact with Bleg1_2337 with lowest energy of $-1163.8 \mathrm{KJ} / \mathrm{mol}$. From the results, Bleg1_2337 could be seen embracing Bleg1_2507 with an extended arm (Figure 5(A)). Refinement of the docked protein complex successfully decreased the potential energy from $-257189.2 \mathrm{KJ} / \mathrm{mol}$ to $-295640.7 \mathrm{KJ} / \mathrm{mol}$ signifying favorable protein conformation.

Inspecting the interface of both interacting proteins, Bleg1_2507 possessed a set of acidic residues such as Asp-47, 55, 56 and 126, Glu-48, 141 and 169 on its surface (Figure 5(B)). This observation lends support to ProtParam analysis which revealed that Bleg1_2507 has an acidic pI of 3.95, indicating this HP has abundant number of acidic residues. Its putative COX partner, Bleg1_2337, on the other hand, possessed a set of positively charged, basic residues on its surface such as Lys3, 34, 69,73, 182 and 206, Arg-8, 71 and 72 and His-215 (Figure $5(\mathrm{C})$ ), which gave the protein a basic $\mathrm{pI}$ value of 4.21 according to ProtParam analysis. The presence of these oppositely charged residues on the respective proteins undoubtedly facilitated ionic interaction between the two macromolecules. Such ionic interaction between Sco and COX proteins has been similarly observed and was proposed to generate a charged-mediated Sco-apo$\mathrm{CuA}$ located in the COX protein which will allow copper exchange to occur [39].

Based on the mechanism proposed for BsSco protein, for copper to be bound and later transferred from Sco to COX, both of the conserved Cysteine residues in the CXXXC motif of BsSco will be firstly reduced to di-thiol state via formation of $\mathrm{SH}-\mathrm{SH}$ bond. This will result in a reduced BsSco protein that is able to strongly interact with $\mathrm{Cu}(\mathrm{II})$ ion to form $\mathrm{BsSco}-\mathrm{Cu}(\mathrm{II})$ complex. From the BsSco-Cu(II) complex, the metal ion will then be transferred to its intended target i.e. apo-subunit II of COX via association of both of the proteins (BsSco and COXII). In this process, the Histidine residue was postulated to play an important role in this second phase of copper coordination by regulating the structural dynamism of bacterial Sco [6]. During association of both of the proteins, apart from metal ion transfer, an oxidationreduction (redox) process also takes place via electron transfer [39]. During interaction of the proteins, BsSco will undergo oxidation by releasing electrons which will 


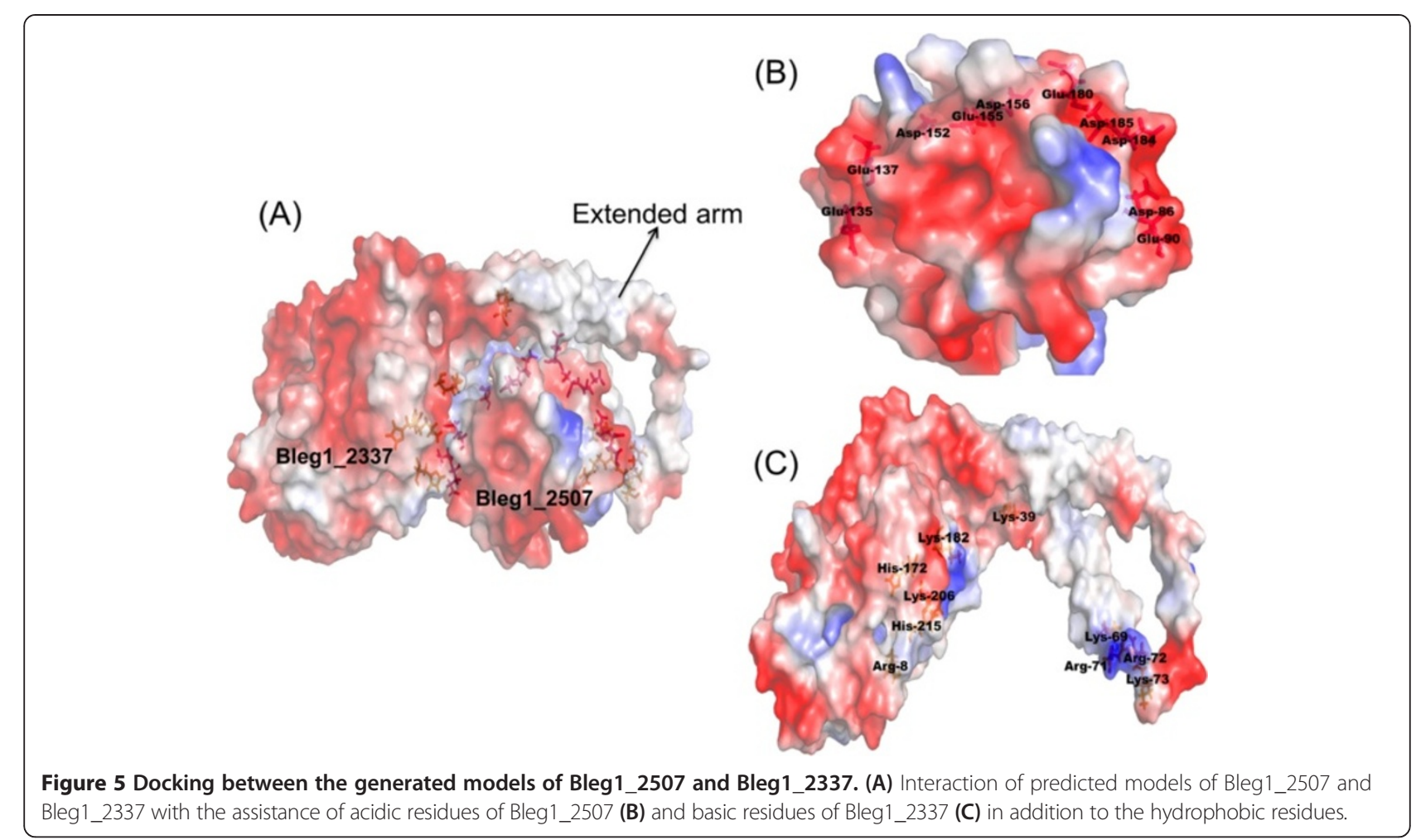

be subsequently delivered to the binuclear $\mathrm{CuA}$ site of COXII. Consequently, the received electrons in the $\mathrm{CuA}$ site of COXII which made up of Cys-217, 221 and His215 will reduce $\mathrm{Fe}(\mathrm{III})$ to $\mathrm{Fe}(\mathrm{II})$ or $\mathrm{Cu}(\mathrm{II})$ to $\mathrm{Cu}(\mathrm{I})$ [39]. The di-thiol state of BsSco is vital in this electron transfer process and maintaining the redox state of the partner.

In the case of Bleg1_2507, it is therefore highly possible that it may possess redox properties by potentially acting as a thiol disulfide oxidoreductase. Similar to BsSco, both the conserved Cys-69 and Cys-73 in Bleg1_2507 may also form the $\mathrm{SH}-\mathrm{SH}$ bond and give forth the di-thiol state of the protein. From this point onwards, Bleg1_2507 HP may be able to undergo oxidation to release electrons which are subsequently delivered to the binuclear $\mathrm{CuA}$ site of reduced COXII of Bleg1_2337 which were made up of Cys-207, 211 and His-215 and Cys-261, 264 and His-265 (highly similar to the other documented COXII proteins), to reduce the respective metal ion at the site. This postulation is further supported by the presence of hydrophobic grooves in HP Bleg1_2507 which form an extensive uncharged surface surrounding the active disulfide bond formed by Cys-69, Cys-73 and His-159 encompassed in a solvent exposed environment which might facilitate the electron transfer process, similar to the features observed in BsSco [35].

It is worthy to note that other than binding metal ions, the conserved Cys pair and His-ligand loops of Sco proteins are important for Sco to interact with other proteins as well [33]. This feature was clearly observed in our docking results where these residues of HP Bleg1_2507 are shown to interact with putative COXII (Bleg1_2337) via hydrophobic interaction, stabilized by uncharged surface features surrounding the hydrophobic pocket between loop 4 and loop 9 (Figure 4(B)). In the Cysteine pair loop (loop 4), it possessed uncharged polar residues such as Thr-67, 70, 71 and 75 and Asn-68. While, Thr-153, 161 and Ser-160 were located in the His-ligand loop (loop 9). Similarly in its redox partner, the putative COXII (Bleg1_2337), crucial hydrophobic residues such as Val-82 and 109, Gly-84, Trp-92 and 175, Ile-95, Pro-96 and 107, Leu-99, Phe-106 and 174, Thr-108, Ser-173 and Pro177 may have contributed to the interaction of both proteins (Figure 4(D)), in accordance to other similarly reported study [39]. All of these residues are crucial in maintaining the intactness of Sco$\mathrm{CuA}$ protein complex for electron transfer [39].

\section{Structural and functional comparison of Sco proteins}

Comparing the sequences of the predicted Sco Bleg1_2507, BsSco (1XZO, chain A), human Sco1 (2GT5, chain A) and yeast Sco1 (2BTK, chain A), these proteins share many conserved residues regardless of their taxonomic and sequence differences (Figure 1). Superimposition of predicted protein model of Bleg1_2507 with the 3D structure of BsSco showed that they are highly similar with RMSD value of $0.286 \AA$ (Figure 6(A)). Interestingly, superimposition of 

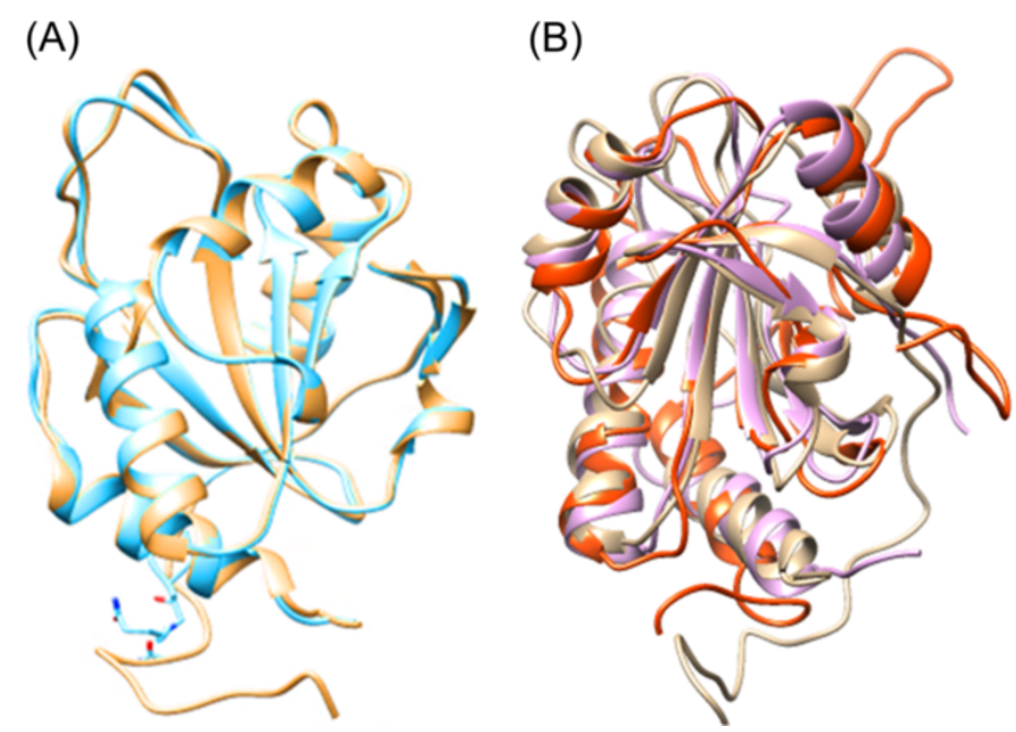

Figure 6 Structural alignment of Bleg1_2507 protein model with BsSco, human and yeast Sco1 proteins. (A) Superimposition of Ca atoms between Bleg1_2507 (brown) and BsSco [1XZO, Chain A] (blue), (B) human Sco1 [2GT5, Chain A] (orange) and yeast Sco1 [2BTK, Chain A] (magenta) proteins exhibited structural resemblance across biological taxa.

predicted protein model of Bleg1_2507 with the 3D structures of human and yeast Scol showed that their structures are comparatively similar as well even at RMSD values of $1.215 \AA$ and $1.060 \AA$ respectively (Figure 6(B)).

It is important to note that Bleg1_2507, BsSco and Scol of yeast contain the same number of $\beta$-sheets and $\alpha$-helices i.e. eight and four respectively. The 3D structure of human Sco1 (2GT5, chain A), however, contained only six $\beta$-sheets. The two additional $\beta$ sheets in Bleg1_2507 are $\beta 2$ and $\beta 3$, which forms the hairpin structure located before the $3_{10^{-}}$- helix at the $\mathrm{N}$-terminus.

In addition, although Bleg1_2507 shares relatively low sequence identity to BsSco (47\%), human Sco1 (2GT5, chain A) (27\%) and yeast Sco1 (27\%), the predicted metal-binding residues of Bleg1_2507 i.e. Cys-69, Cys-73 and His-159, were located at flexible active loops (loop 4 and 9) similar to other Sco proteins across biological taxa.

It is indeed interesting to note that despite their remarkable structural similarity, Sco proteins are rather diverse in function. In eukaryotes, Sco proteins are involved in the assembly of the $\mathrm{CuA}$ cofactor of mitochondrial cytochrome c oxidase, redox signaling and regulation of copper homeostasis [13]. In prokaryotes, Sco proteins are more promiscuous in functions. While some are required for COX biosynthesis, others have been implicated in different processes such as copper delivery to other enzymes and protection against oxidative stress [33]. More investigations as to the physiological function and mechanism of Sco proteins will be useful in determining its exact role and importance in the system biology of an organism.

\section{Conclusions}

In this study, genome mining of hypothetical protein sequences of $B$. lehensis $\mathrm{G} 1$ alkaliphile led to the discovery of Bleg1_2507 which showed the presence of a Trx-like domain linked to Sco protein. Showing only $35 \%$ of similarity to BsSco, the prokaryotic model of Sco, the 3D model of Bleg1_2507 was built by homology modeling. The built protein model showed good preservation of the $\beta \alpha \beta \alpha \beta \beta$ core structure (characteristic of many Trx-like redox proteins), three flanking $\beta$-sheets, a $3_{10}-$ helix at the N-terminus and a hairpin (characteristic of Sco proteins). Unique to Bleg1_2507, there is an $\alpha$-helix $(\alpha 2)$ near loop 4 and a $\beta$-strand ( $\beta 6)$ that are parallel with $\beta 5$ and $\beta 4$, which are not present in Trx. Another distinct feature of Bleg1_2507 compared to BsSco is the difference in the location of the metal-binding ligands in Bleg1_2507. Docking simulations interestingly provided a view of Bleg1_2507 in association with its putative COXII redox partner, Bleg1_2337, where the latter can be seen to hold its partner in an embrace, facilitated via hydrophobic and ionic interaction between the two proteins. Although Bleg1_2507 shares relatively low sequence homology to BsSco, interestingly, it is structurally similar to the protein as well as other Sco proteins across biological taxa. This observation highlights that despite its varying functions in prokaryotes and eukaryotes, Sco proteins are structurally well preserved.

Based on all the results obtained, we hereby propose that HP Bleg1_2507 of B. lehensis G1 is a Sco protein which is able to interact with COXII, its redox partner. Hence, it is highly possible that HP Bleg1_2507 may possess metallochaperone and disulfide redox functions 
similar to other documented bacterial Sco proteins. It is hoped that the predicted structure and function of Bleg1_2507 from the hypothetical protein dataset of $B$. lehensis G1 will help to spur the search for other physiologically relevant proteins among the so-called "orphan" proteins of any given organism as well as direct future biological studies in improving our understanding of COX complex assembly process.

\section{Competing interests}

The authors declare that they have no competing interests.

\section{Authors' contributions}

TSH carried out bioinformatics analyses and clustering of all the hypothetical proteins, homology modelling and protein docking in this study. TSH and YMN drafted the manuscript. ABS, MBAR, RAK, ALTC, AMAM, NMM revised and proofread the manuscript. YMN conceived the study and participated in its design and coordination together with ABS, MBAR. RAK, ALTC, AMAM gave technical advice to the study. All authors read and approved the final manuscript.

\section{Acknowledgement}

We would like to thank Mr. Yusuf Muhammad Noor of Malaysia Genome Institute (MGI) in providing the initial genome information, Ministry of Science, Technology and Innovation for Sciencefund, MOSTI-MGI for Sciencefund Special Allocation (Project code: 02-05-20-SF11112) and facility, Universiti Putra Malaysia (UPM) for Graduate Research Fellowship awarded to Ms. Tan Soo Huei.

\section{Author details}

${ }^{1}$ Center for Enzyme and Microbial Biotechnology (EMTECH), Faculty of Biotechnology and Biomolecular Sciences, Universiti Putra Malaysia, Serdang, Selangor 43400, Malaysia. ²Department of Chemistry, Faculty of Science, Universiti Putra Malaysia, Serdang, Selangor 43400, Malaysia. ${ }^{3}$ School of Biosciences and Biotechnology, Faculty of Science and Technology, Universiti Kebangsaan Malaysia, 43600 UKM, Bangi, Selangor, Malaysia. ${ }^{4}$ Malaysia Genome Institute, Ministry of Science, Technology and Innovation, Jalan Bangi, Kajang, Selangor 43000, Malaysia.

Received: 13 September 2013 Accepted: 14 March 2014 Published: 19 March 2014

\section{References}

1. Bork P: Powers and pitfalls in sequence analysis: the $70 \%$ hurdle. Genome Res 2000, 10(4):398-400.

2. Galperin MY, Koonin EV: 'Conserved hypothetical' proteins: prioritization of targets for experimental study. Nucl Acid Res 2004, 32(18):5452-5463.

3. Roberts RJ: Identifying Protein Function- A Call for Community Action. PLoS Biol 2004, 2(3):e42.

4. Desler C, Suravajhala P, Sanderhoff M, Rasmussen M, Rasmussen LJ: In Silico screening for functional candidates amongst hypothetical proteins. BMC Bioinforma 2009, 10(289):1471-2105.

5. Samsulrizal NH, Abdul Murad AM, Noor YM, Jema'on NA, Najimudin N, Illias RM, Abu Bakar FD, Mahadi NM: Genome Sequencing and Annotation of Alkaliphilic Bacterium Bacillus lehensis G1. India: The Eighth Asia Pacific Bioinformatics Conference, Bangalore (APBC 2010); 2010:18-21.

6. Balatri E, Banci L, Bertini I, Cantini F, Baffoni-Ciofi S: Solution Structure of Sco1: A Thioredoxin-like Protein Invovled in Cytochrome c Oxidase Assembly. Structure 2003, 11(11):1431-1443.

7. Glerum DM, Shtanko A, Tzagoloff A: Characterization of COX17, a yeast gene involved in copper metabolism and assembly of cytochrome oxidase. J Biol Chem 2003, 271(24):14504-14509.

8. Krummeck G, Rödel G: Yeast Sco1 protein is required for a posttranslational step in the accumulation of mitochondrial cytochrome c oxidase subunits I and II. Curr Genet 1990, 18(1):13-15.

9. Schulze M, Rödel G: SCO1, a yeast nuclear gene essential for accumulation of mitochondrial cytochrome c oxidase subunit II. Mol Gen Genet 1988, 211(3):492-498.
10. Abajian C, Rosenzweig CA: Crystal structure of yeast Sco1. J Biol Inorg Chem 2006, 11(4):459-466.

11. Papadopoulou LC, Sue CM, Davidson MM, Tanji K, Nishino I, Sadlock JE, Krishna S, Walker W, Selby J, Glerum DM, Coster RV, Lyon G, Scalais E, Lebe R, Kaplan P, Shanske S, De Vivo DC, Bonilla E, Hirano M, DiMauro S, Schon EA: Fatal infantile cardioencephalomyopathy with COX deficiency and mutations in SCO2, a COX assembly gene. Nat Genet 1999, 23(3):333-337.

12. Attallah CV, Welchen E, Martin AP, Spinelli SV, Bonnard G, Palatnik JF, Gonzalez DH: Plants contain two SCO proteins that are differentially involved in cytochrome c oxidase function and copper and redox homeostasis. J Exp Bol 2011, 62(12):4281-4294.

13. Valnot I, Osmond S, Gigarel N, Mehaye B, Amiel J, Cormier-Daire V, Munnich A, Bonnefont JP, Rustin P, Rötig A: Mutations of the SCO1 gene in mitochondrial cytochrome $c$ oxidase deficiency with neonatal-onset hepatic failure and encephalopathy. Am J Hum Genet 2000, 67(5):1104-1109.

14. Quevillon E, Silventoinen V, Pillai NS, Harte N, Mulder N, Apweile R, Lopez R: InterProScan: protein domains identifier. Nucl Acids Res 2005, 33(Web Server Issue):W116-W120

15. Marchler-Bauer A, Anderson JB, Derbyshire MK, DeWeese SC, Gonzales NR, Gwadz M, Hao L, He S, Hurwitz DI, Jackson JD, Ke Z, Krylov D, Lanczycki CJ, Liebert CA, Liu C, Lu F, Lu S, Marchler GH, Mullokandov M, Song JS, Thanki N, Yamashita RA, Yin JJ, Zhang D, Bryant: CDD: a conserved domains and protein three-dimensional structure. Nucl Acids Res 2013, 41(D1):D348-D352.

16. Altschul SF, Madden TL, Schäffer AA, Zhang J, Zhang Z, Miller W, Lipman DJ: Gapped-BLAST and PSI-BLAST: a new generation of protein database search programs. Nucl Acids Res 1997, 25(17):3389-3402.

17. De Castro E, Sigrist CJA, Gattiker A, Bulliard V, Langendijk-Genevaux PS, Gasteiger E, Bairoch A, Hulo N: ScanProsite: detection of PROSITE signature matches and ProRule-associated functional and structural residues in proteins. Nucl Acids Res 2006, 34(Web Server Issue):W362-W365.

18. Ashkenazy H, Erez E, Martz E, Pupko T, Ben TN: ConSurf 2010: calculating evolutionary conservation in sequence and structure of proteins and nucleic acids. Nucl Acids Res 2010, 38(Suppl 2):W529-W533.

19. Passerini A, Lippi M, Frasconi P: MetalDetector v2.0: predicting the geometry of metal binding sites from protein sequence. Nucl Acids Res 2011, 39(Suppl 2):W288-W292.

20. Gasteiger E, Hoogland C, Gattiker A, Duvaud S, Wilkins MR, Appel RD Bairoch A: Protein Identification and Analysis Tools on the ExPASy Server. In The Proteomics Protocols Handbook. Edited by John MW. Totowa, New Jersey, USA: Humana Press; 2005:571-607.

21. Rice $P$, Longen $T$, Bleasby A: The European Molecular Biology Open Software Suite. Trends Genet 2000, 16(6):276-277.

22. Felsenstein J: PHYLIP - Phylogeny Inference Package (Version 3.2). Cladistics 1989, 5:164-166.

23. Larkin MA, Blackshields G, Brown NP, Chenna R, McGettigan PA, McWilliam H, Valentin F, Wallance IM, Wilm A, Lopez R, Thompson JD, Gibson TJ, Higgins DG: Clustal W and Clustal X version 2.0. Bioinformatics 2007, 23(21):2947-2948.

24. Wali A, Blundell TL: Comparative protein modeling by satisfaction of spatial restraints. J Mol Biol 1993, 234(3):779-815.

25. Delano WL: The PyMOL Molecular Graphics System. San Carlos, CA, USA: Delano Scientific; 2002

26. Kojetin DJ, Thompson RJ, Cavanagh J: Hypothesis: Sub-classification of response regulators using the surface characteristics of their receiver domains. FEBS Lett 2004, 5601(1-3):227-228.

27. Pettersen EF, Goddard TD, Huang CC, Couch GS, Greenblatt DM, Meng EC, Ferrin TE: UCSF Chimera-a visualization system for exploratory research and analysis. J Comput Chem 2004, 25(13):1605-1612.

28. Schymkowitz JW, Rousseau F, Martins IC, Ferkinghoff-Berg J, Stricher F, Serrano L: Prediction of water and metal-binding sites and their affinities by using the Fold-X force field. Proc Natl Acad Sci USA 2005, 102 (29):10147-10152.

29. Colovos C, Yeates OT: Verification of protein structures: Patterns of nonbonded atomic interactions. Prot Sci 1993, 2(9):1511-1519.

30. Laskoswki RA, MacArthur MW, Moss DS, Thornton JM: PROCHECK: a program to check the stereochemical quality of protein structures. J Appl Cryst 1993, 26(2):283-291.

31. Kozakov D, Hall DR, Beglov D, Brenke R, Comeau SR, Shen Y, Keyong L, Jiefu Z, Vakili P, Paschalidis Cl, Vajda S: Achieving reliability and high accuracy in automated protein docking: ClusPro, PIPER, SDU, and stability analysis in CAPRI rounds 13-19. Proteins 2004, 78:3124-3130. 
32. Krieger E, Joo K, Lee J, Raman S, Thompson J, Tyka M, Baker D, Karplus L: Improving physical realism, stereochemistry, and side-chain accuracy in homology modeling: Four approaches that performed well in CASP8. Proteins 2009, 77(Suppl 9):114-122.

33. Banci L, Bertini I, Cavallaro G, Baffoni-Ciofi S: Seeking the determinants of the elusive functions of Sco proteins. FEBS J 2011, 278(13):2244-2262.

34. Blundell KL, Wilson MT, Svistunenko DA, Vijgenboom E, Worrall JA: Morphological development and cytochrome c oxidase activity in Streptomyces lividans are dependent on the action of a copper bound Sco protein. Open Biol 2013, 3(1):120163.

35. Banci L, Bertini I, Cavallaro G, Rosato A: The Functions of Sco Proteins from Genome-Based Analysis. J Proteome Res 2007, 6(4):1568-1579.

36. Lyons JA, Aragao D, Slattery O, Pisliakov AV, Soulimane T, Caffrey M: Structural insights into electron transfer in caa3-type cytochrome oxidase. Nature 2012, 487(7408):514-518.

37. Ye Q, Iveta SI, Hill BC, Chao JZ: Identification of a Disulfide Switch in BsSco, a Member of the Sco Family of Cytochrome c Oxidase Assembly Proteins. Biochemistry 2005, 44(14):5552-5560.

38. Kinch LN, Baker D, Grishin NV: Deciphering a Novel Thioredoxin-Like Fold Family. Proteins 2003, 52(3):323-331.

39. Banci L, Bertini I, Baffoni-Ciofi S, Kozyreva T, Mori M, Wang S: Sco proteins are involved in electron transfer processes. J Biol Inorg Chem 2011, 16(3):391-403.

40. Banci L, Bertini I, Calderone V, Ciofi-Baffoni S, Mangani S, Martinelli M, Palumaa P, Wang S: A hint for the function of human Scol from different structures. Proc Natl Acad Sci USA 2006, 103:8595-8600.

41. Andruzzi L, Nakano M, Nilges Mark J, Blackburn Ninian J: Spectroscopic Studies of Metal-Binding and Metal Selectivity in Bacillus subtilis BSco, a Homologue of the Yeast Mitochondrial Protein Sco1p. J Am Chem Soc 2005, 127(47):16548-16558.

doi:10.1186/1472-6807-14-11

Cite this article as: Tan et al: A Sco protein among the hypothetical proteins of Bacillus lehensis G1: Its 3D macromolecular structure and association with Cytochrome C Oxidase. BMC Structural Biology 2014 14:11

\section{Submit your next manuscript to BioMed Central and take full advantage of:}

- Convenient online submission

- Thorough peer review

- No space constraints or color figure charges

- Immediate publication on acceptance

- Inclusion in PubMed, CAS, Scopus and Google Scholar

- Research which is freely available for redistribution 